\title{
Intravascular Papillary Endothelial Hyperplasia (Masson's Tumour) Arising from The Superior Sagittal Sinus Masquerading as an Aggressive Malignant Lesion: A Rare Case Report
}

\author{
K V Vinu Balraam, Reena Bharadwaj and Sampath K S
}

Department of Pathology, Armed Forces Medical College, Off Sholapur Road, Pune, Maharashtra. India

\begin{abstract}
Intravascular papillary endothelial hyperplasia (Masson's Tumour) is an infrequently occurring reactive vascular lesion which is typified by papillary intravascular proliferation of endothelial cells. It was first described by Masson in his publication in the year 1923. It usually arises in the extra-cranial location ordinarily in the skin and subcutaneous soft tissue. They are extremely uncommon in the intra-cranial sites with only a handful of cases reported in the literature amongst more than 300 cases of the same lesion reported in the sites arising outside the cranium. The authors here would like to report a rare case of Masson's tumour arising from the superior sagittal sinus eroding the skull bone and ultimately leading to an oval defect in the skull. The one-off nature of this location is what is intriguing in this case.
\end{abstract}

Keywords: Intracranial; Intravascular Papillary Endothelial Hyperplasia; Masosn's Tumour; Superior Sagittal Sinus.

\section{Introduction}

Intravascular papillary endothelial hyperplasia (IPEH), commonly known as Masson's Tumour is a reactive vascular proliferation which usually denotes a strange form of organising thrombus (1). This lesion typically presents in three forms: 1. Primary - Pure form involving an isolated dilated blood vessel; 2 . Secondary - Focal change in a pre-existing vascular lesion; 3. Extra-vascular site in association with haematoma. Location wise they can be intracranial and extracranial, latter being the commonest. Outside the cranial cavity, they commonly occur in the skin and subcutis of the extremities, head and neck, trunk and in the visceral organs. Intracranial location in the skull base, sella, petrous part of temporal bone and a solitary case in the pineal region has been mentioned in the literature. Almost over 300 cases of Masson's tumour have been reported in the literature, but only a handful are intracranial in location and no case has been reported to be arising from the superior sagittal sinus. Intracranial cases usually come to notice due to their pressure effects. Here, we report a rare case of IPEH arising from the superior sagittal sinus and eroding the skull bone ultimately leading to an oval defect in the skull.

\section{Case Report}

A 39 years old male with no known co-morbidities, presented to the surgical OPD of our health care setup with progressive swelling over the midline of scalp with no associated symptoms of headache, vomiting, seizures or loss of consciousness or past history of trauma. The patient was taken up for excision under local anaesthesia with a diagnosis of sebaceous cyst. However, the procedure was abandoned due to excessive bleeding and only a biopsy was taken which was reported as arterio-venous malformation. The patient was then referred to the department of neurosurgery, where on examination they felt a $10 \times 10 \mathrm{~cm}$ soft, non-tender, non-compressible and non-pulsatile swelling in the midline fronto-parietal region with no bruit. Systemic examination was unremarkable. Preoperative MRI revealed a left parasagittal heterogeneous high parietal extraaxial, extracranial and intracranial lesion measuring $5.3 \times 5 \times 4.5 \mathrm{~cm}$ with a post-contrast enhancement. The differentials offered were: Atypical meningioma, hemangiopericytoma, bony tumour or a metastatic tumour. The patient underwent bi-frontal craniotomy and enbloc removal of the involved bone with the aneurysmal segment of the superior sagittal sinus. The excised segment was received for histopathological examination at our laboratory. Grossly, an elliptical craniotomy specimen was received with adequate clear margins on all sides, comprising of dural base along with the superior sagittal sinus, parietal bone with a defect in the centre and overlying scalp tissue. A dark brown, spongy haemorrhagic tumour, measuring $8 \times 7 \times 4.5 \mathrm{~cm}$ was seen to arise from the superior sagittal sinus, breaching the parietal bone and entering the sub-galeal region of the overlying scalp (Figure 1). Sections from the tissue submitted revealed a highly vascular lesion arising from the endothelial lining of a large vessel attached to the dura. The lesion, confined to the intra-vascular space, 
was arranged in papillary architecture lined by flattened endothelial cells with a central hyalinised core (Figure 2). The cells were bland without any noticeable atypia or mitosis. Large areas of organising thrombus was noted along with haemorrhage within the lesion. These endothelial cells

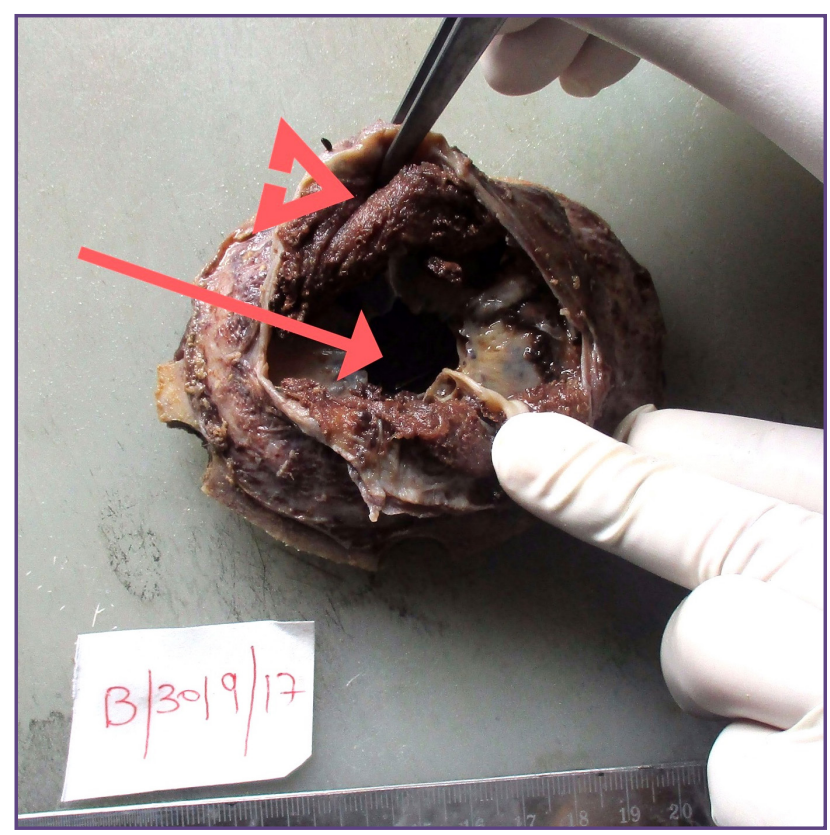

Fig. 1: Gross specimen showing the tumour (arrow head) with the attached dura (forceps) breaching the parietal bone and creating an oval defect in the bone (arrow). were highlighted by CD31 and CD34 (Figure 3). MIB-1 labelling index was imperceptible (less than 1\%). Based on these histomorphological and immunohistochemical features, a final sign out of Intravascular Papillary Endothelial Hyperplasia (Masson's Tumour) was made.

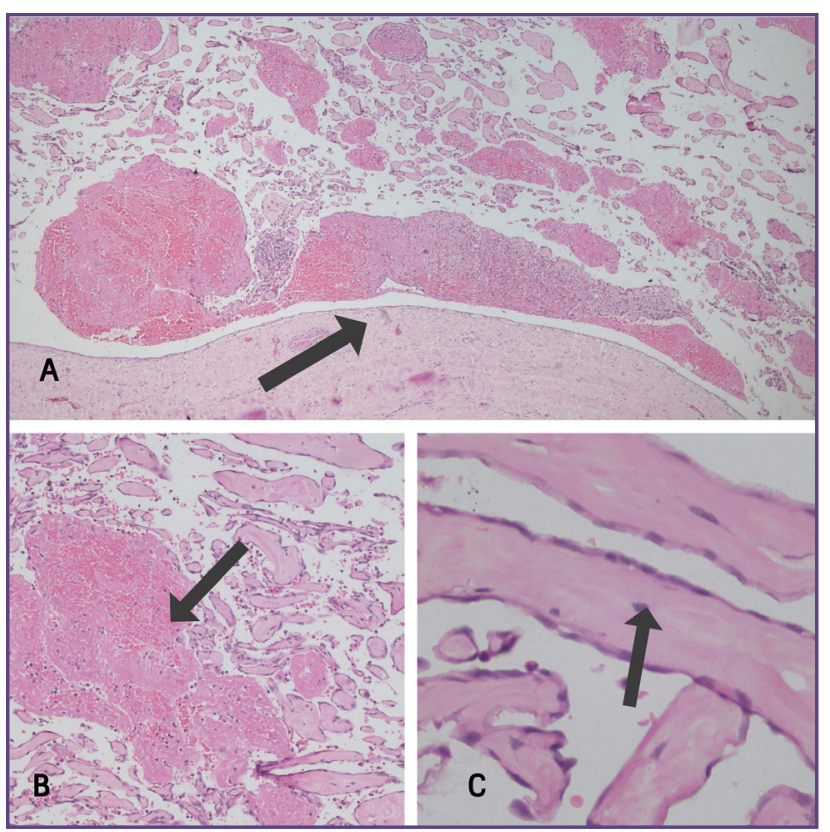

Figure 2: (Haematoxylin \& Eosin Stain), A - Lesion seen arising from the vessel wall (arrow) (40x)., B - Areas of organizing thrombus (arrow) with surrounding hyalinised vascular cores $(100 x)$., $C$ - Hyalinised vascular cores (arrow) lined by endothelial cells (400x).

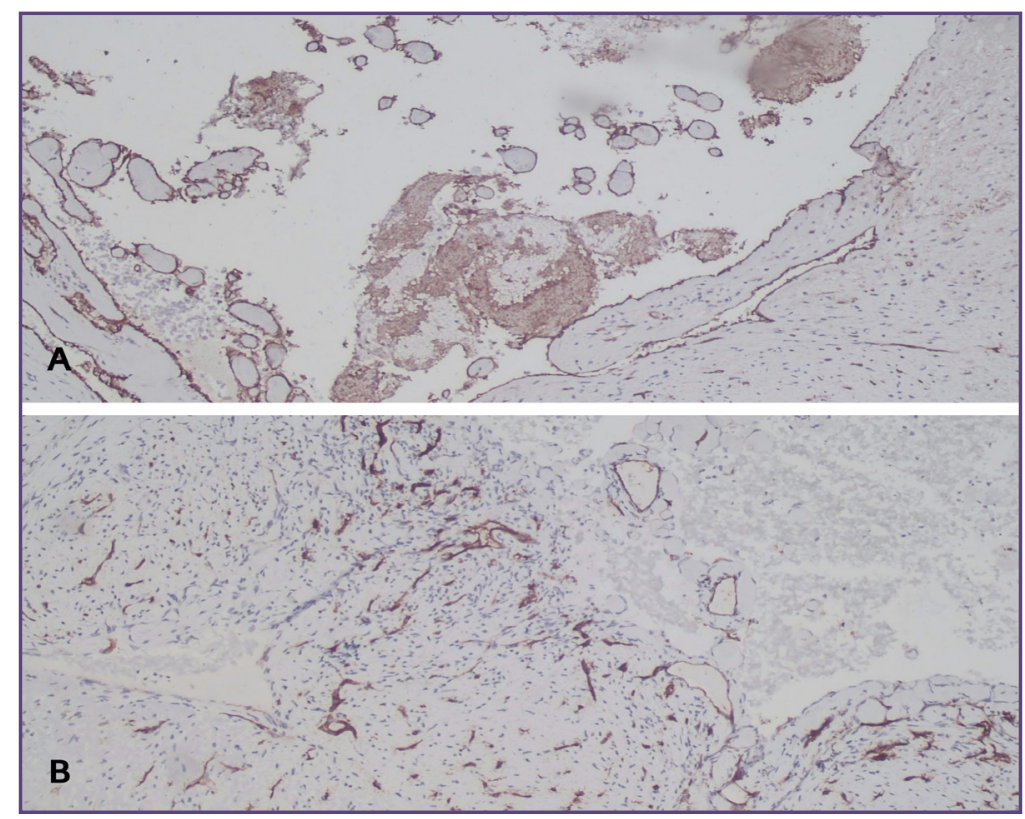

Fig. 3: Hyalinised vascular cores lined by endothelial cells which are highlighted by CD31 (3A) and CD34 (3B) immunostains (Immunohistochemistry using 3, 3'-diaminobenzidine as chromogen; 100x). 


\section{Discussion}

IPEH is a unique reactive non neoplastic condition wherein there is an excessive proliferation of endothelial cells in the affected blood vessel lining. It was first described by Masson in his publication in the year 1923 (2). The evolution of this disease still remains a mystery but many believe it to be an unusual form of organizing thrombus (3). A minority of researchers also think it as a neoplastic process due to endothelial proliferation leading to papillae formation in the lumen and subsequent necrosis as in a red infarct (4).

Throughout the literature, it has been termed as benign and most of them get cured by a simple wide local excision. Till date, only around 30 cases of IPEH have been reported which are intra-cranial in location (5-6). No case of Masson's Tumour has been reported arising from the superior sagittal sinus aggressive enough to erode the overlying parietal bone to create an elliptical defect and protruded out on to the scalp confusing the surgeon initially to be a sebaceous cyst. IPEH shows a marked similarity to a well differentiated angiosarcoma. Hence, it must be excluded with careful judgement. Intracranial IPEH is thought to be usually arising from pre-existing lesions like aneurysm, thrombi or haemangiomas. Thrombus acts as an inciting agent for endothelial cell proliferation in majority of cases while in some cases is a causal effect of IPEH itself. IPEH in the intracranial region has been described in the skull base, brain parenchyma, pineal region, and in the pertrous part of the temporal bone. Even there have been case reports of IPEH of sellar and para-sellar region $(3,7)$. To our best of knowledge, this report is the first of its kind to be originating in the superior sagittal sinus. The imaging findings in IPEH are usually inconspicuous. Histopathology forms an irreplaceable tool for confirming or refuting the diagnosis. Of the previously treated intracranial IPEH cases, excision with wide margins resulted in cure in 10 cases. Remaining cases underwent subtotal resection with a fairly significant recurrence rate. As a consequence, most authors have advocated adjuvant therapy in cases of incomplete resection, mainly in the form of radiotherapy, gamma knife surgery or even chemotherapy (6). Some researchers have also hypothesised that previous radiotherapy may lead to occurrence of IPEH due to blood flow obstruction and thrombus formation (8).

\section{Conclusion}

IPEH is a reactive process which is clinically important as it may present as a mass lesion. Intracranial IPEH itself is an extremely rare entity which needs to be kept in the back of our mind as a differential of any vascular lesion, more so in cases of pre-existing vascular anomalies. This pathological process needs to be meticulously distinguished from angiosarcoma because in almost all the cases, a simple wide local excision is curative enough for IPEH.

\section{Acknowledgements}

The authors would like to acknowledge the lab technicians and the support staff for the effort put in through-out commencing from grossing of the specimen right till the histopathological sections were processed.

\section{References}

1. Kuo T, Sayers CP, Rosai J. Masson's 'vegetant intravascular hemangioendothelioma': a lesion often mistaken for angiosarcoma: study of seventeen cases located in the skin and soft tissues. Cancer. 1976; 38(3):1227-1236.

2. Masson, P.: Hemangioendotheliome vegetant intravasculaire. Bull Soc Anat Paris. 1923; 93: 517-523.

3. Charalambous LT, Penumaka A, Komisarow JM, Hemmerich AC, Cummings TJ, Codd PJ, et al. Masson's tumor of the pineal region: case report. J Neurosurg. 2017 Aug 4;1-6.

4. Bologna-Molina R, Amezcua-Rosas G, Guardado-Luevanos I, Mendoza-Roaf P, González-Montemayor T, MolinaFrechero N. Intravascular Papillary Endothelial Hyperplasia (Masson's Tumor) of the Mouth - A Case Report. Case Reports in Dermatology. 2010; 2(1): 22-26.

5. Duong DH, Scoones DJ, Bates D, Sengupta RP: Multiple intracerebral intravascular papillary endothelial hyperplasia. Acta Neurochir. 1997; 139:883-886.

6. Sim SY, Lim YC, Won KS, Cho KG: Thirteen-year follow-up of parasellar intravascular papillary endothelial hyperplasia successfully treated by surgical excision: case report. J Neurosurg Pediatr. 2015; 15:384-39.

7. Cagli S, Oktar N, Dalbasti T, Işlekel S, Demirtaş E, Ozdamar $\mathrm{N}$ : Intravascular papillary endothelial hyperplasia of the central nervous system-four case reports. Neurol Med Chir. 2004; 44:302-310.

8. Crocker M, deSouza R, Epaliyanage P, Bodi I, Deasy N, Selway R: Masson's tumour in the right parietal lobe after stereotactic radiosurgery for cerebellar AVM: case report and review. Clin Neurol Neurosurg. 2007; 109:811-815.

*Corresponding author:

Dr K V Vinu Balraam, Department of Pathology, Armed Forces Medical College,Off Sholapur Road, Pune - 411040,Maharashtra,India

Phone: +91 7798169619

Email: vbalraam@gmail.com

Financial or other Competing Interests: None. 